\title{
RESPOSTA SOROLÓGICA DE COELHOS IMUNIZADOS COM ANTÍGENOS DE PYTHIUM INSIDIOSUM ASSOCIADOS A DIFERENTES ADJUVANTES
}

\author{
SEROLOGICAL RESPONSE IN RABBITS IMMUNIZED WITH \\ PYTHIUM INSIDIOSUM ANTIGENS ASSOCIATED \\ WITH DIFFERENT ADJUVANTS
}

\author{
Alexandre Trindade Leal ${ }^{1}$ Janio Morais Santurio ${ }^{2}$ Adriana Bardemaker Monteiro Leal ${ }^{1}$ \\ Alexandre Machado Pinto ${ }^{3}$ Josiane Griebeler ${ }^{3}$ Eduardo Furtado Flores ${ }^{4}$ \\ Laerte Ferreiro ${ }^{5}$ João Batista Catto ${ }^{6}$
}

RESUMO

O Pythium insidiosum é um fungo zoospórico que se desenvolve em locais alagadiços e que pode infectar humanos $e$ animais, principalmente eqüinos. A infecção natural nesta espécie resulta em pitiose clínica, uma doença granulomatosa de difícil tratamento. Uma das opções terapêuticas é a imunoterapia com antígenos obtidos de culturas do agente. Com o objetivo de avaliar o efeito de adjuvantes na resposta sorológica a antígenos do $\boldsymbol{P}$. insidiosum, 24 coelhos divididos em 4 grupos foram imunizados com antígeno macerado de micélio (AMM) associado a três adjuvantes. Grupo I: hidróxido de alumínio; grupo II: adjuvante de Freund; grupo III: óleo mineral e grupo IV: água destilada -controle. Os tratamentos foram avaliados a diferentes intervalos, quanto à capacidade de induzir a produção de imunoglobulinas específicas da classe G, através da técnica de ELISA. Na fase 1, os animais receberam três doses do imunógeno (dias zero, 14 e 28) e foram avaliados sorologicamente nos dias 14, 21, 28 e 35. Nessa fase, os adjuvantes oleosos (GII e III) induziram niveis de anticorpos estatisticamente superiores aos induzidos nos grupos I e IV. Durante a fase 2 (dias 42 a 120), cada grupo foi subdividido em dois; sendo um subgrupo mantido em tratamento (imunizações adicionais nos dias 42, 56, 68 e 82) $e$ o outro tendo o tratamento interrompido após a $3^{\underline{a}}$ dose (dia 28). Nos subgrupos mantidos em tratamento, os níveis de anticorpos dos grupos imunizados com adjuvantes foram estatisticamente superiores aos induzidos no grupo GIV (controle). Nos coelhos com tratamento interrompido, os grupos I, II e III apresentaram

\begin{abstract}
manutenção nos níveis de $\operatorname{IgG}$ e foram estatisticamente superiores ao grupo controle, que apresentou declínio nos níveis de anticorpos. Os resultados demonstraram a capacidade dos adjuvantes testados em potencializar e prolongar a resposta humoral aos antígenos do P. insidiosum. O uso de adjuvantes associado aos atuais imunógenos pode aumentar os índices de cura em eqüinos submetidos à imunoterapia, assim como viabilizar sua utilização como preventivo.
\end{abstract}

Palavras-chave: Pythium insidiosum, imunoterapia, adjuvantes, coelhos, ELISA.

\section{SUMMARY}

Pythium insidiosum is a zoosporic fungi living in flooded areas which can infect humans and animals. Natural infection in these species results in clinical pythiosis, a granulomatous disease of difficult treatment. Immunotherapy with antigens obtained from cultures of the agent is a promising alternative therapy. In order to evaluate the effect of adjuvants in the immunologic response to $\boldsymbol{P}$. insidiosum antigens, 24 rabbits were assigned to four groups and immunized with mycelian mass antigen with each of there adjuvants. Group I: aluminum hydroxide; group II: Freund's adjuvant; group III: mineral oil and group IV: distilled water-control. The effects of the adjavants were evaluated by measuring the levels of anti-pythium imunoglobulin $G(\operatorname{Ig} G)$ produced by the immunized rabbits at different time-points after immunization, using an ELISA test.

${ }^{1}$ Médico Veterinário, Laboratório de Pesquisas Micológicas (LAPEMI), Departamento de Microbiologia e Parasitologia, Universidade Federal de Santa Maria (UFSM).

${ }^{2}$ Médico Veterinário, Professor do Departamento de Medicina Veterinária Preventiva (DMVP), UFSM, 97105-900, Santa Maria, RS. Email: santurio@ccr.ufsm.br (autor para correspondência).

${ }^{3}$ Acadêmico de Veterinária, UFSM.

${ }^{4}$ Médico Veterinário, Professor do DMVP e Departamento de Microbiologia e Parasitologia, UFSM.

${ }^{5}$ Médico Veterinário, Professor da Faculdade de Veterinária, Universidade Federal do Rio Grande do Sul.

${ }^{6}$ Médico Veterinário - CNPGC - EMBRAPA - Campo Grande, MS. 
During phase 1, the animals were immunized three times (days zero, 14 and 28) and serologically tested at days 14, 21, 28 and 35. The oil adjuvants (groups II and III) were statistically superior to groups I and IV. During phase 2 (from day 42 to 120) each group was subdivided in two, with one subgroup having additional immunizations at days 42, 56, 68 and 82 and the other having the treatment interrupted. Among the rabbits with continued immunizations, groups I, II and III (adjuvants) had statistically higher IgG levels than GIV. Among rabbits with interrupted treatment, GI, GII and presented stable IgG levels and were statistically superior to the control group, that presented decrease in the levels. These results demonstrated that the adjuvants were capable of inducing stronger and longer imunologic responses $(\mathrm{Ig} G)$ to $\boldsymbol{P}$. insidiosum antigens. Therefore, the use of adjuvants associated with $\boldsymbol{P}$. insidiosum antigens may increase the recovery rates obtained through immunotherapy.

Key words: Pythium insidiosum, immunotherapy, adjuvants, rabbits.

\section{INTRODUÇÃO}

A pitiose é uma doença granulomatosa que atinge eqüinos, caninos, bovinos, felinos e humanos e ocorre em áreas tropicais, subtropicais e temperadas (MEIRELES et al., 1993; MENDOZA et al., 1996). A espécie eqüina é a mais atingida, principalmente nas formas cutânea e subcutânea, além da forma gastrointestinal (CHAFFIN et al., 1995). Nessa espécie, a doença caracteriza-se pela formação de granulomas eosinofílicos, com a presença de massas necróticas chamadas de kunkers (MENDOZA \& ALFARO, 1986; MEIRELES et al., 1993). No Brasil, a pitiose já foi descrita em eqüinos, bovinos e caninos. A maioria dos casos refere-se a lesões cutâneas em eqüinos, sendo que os relatos publicados já somam mais de 90 casos, distribuídos por várias regiões do país. O Pantanal brasileiro destaca-se como local de maior ocorrência da pitiose eqüina (LEAL $\boldsymbol{e t}$ al., 2001). O agente etiológico - Pythium insidiosum é um oomiceto pertencente ao reino Stramenopila (ALEXOPOULOS $\boldsymbol{e t} \boldsymbol{a l} .$, 1996) e caracteriza-se pela ausência de quitina na parede celular e ausência de esteróides na membrana plasmática. Essas características dificultam a ação das drogas antifúngicas e representam uma grande dificuldade para o tratamento da pitiose (FOIL, 1996).

Os resultados obtidos com as drogas antifúngicas no tratamento da pitiose têm sido variáveis. As drogas mais utilizadas até o presente momento foram a anfotericina B, cetoconazol, miconazol, fluconazol e itraconazol, além dos compostos iodínicos como iodeto de potássio e sódio (LEAL $\boldsymbol{e t}$ al., 2001). A ausência de ergosterol (alvo da ação da maioria dos antifúngicos) na membrana celular do $\boldsymbol{P}$. insidiosum é a principal causa de insucesso no tratamento (FOIL, 1996). O tratamento cirúrgico requer a excisão de toda área afetada, porém isso é dificultado pelas estruturas anatômicas envolvidas, principalmente nos membros (MILLER, 1981). A eficiência de coelhos como modelo experimental para estudo da pitiose foi demonstrado por MILLER \& CAMPBELL (1983).

A imunoterapia tem demonstrado ser um método viável para o tratamento dessa enfermidade, embora os mecanismos imunológicos envolvidos na cura ainda não sejam conhecidos (MENDOZA $\boldsymbol{e t}$ al., 1996). Esse método foi inicialmente proposto por MILLER (1981), que desenvolveu um imunobiológico a partir de culturas do agente. Posteriormente, outros pesquisadores desenvolveram diferentes métodos para produção do imunoterápico, sempre visando à obtenção de antígenos capazes de desencadear uma resposta imunológica eficiente (MILLER \& CAMPBELL, 1982; MENDOZA \& ALFARO, 1986; MENDOZA $\boldsymbol{e} t$ al., 1992a; MONTEIRO, 1999). Os índices de cura dos imunoterápicos para a pitiose eqüina têm sido variáveis. No Brasil, MONTEIRO (1999) obteve entre 50 e $83 \%$ de recuperação clínica.

A utilização de substâncias capazes de potencializar a resposta imunológica, classicamente definidas como adjuvantes, representa uma importante ferramenta para melhorar a eficiência de imunizações com antígenos específicos (KLEIN \& HOREJSI, 1997). O potencial dos adjuvantes difere significativamente de acordo com o antígeno utilizado. Eles podem modificar a conformação ou alterar a carga elétrica da molécula do antígeno, alterando a sua imunogenicidade (VANSELOW, 1987). Com relação à imunoterapia da pitiose, o uso de adjuvantes associado ao imunoterápico pode aumentar os índices de cura, assim como viabilizar o seu uso como preventivo.

O objetivo deste estudo foi avaliar o efeito de três adjuvantes associados a antígenos do $\boldsymbol{P}$. insidiosum na indução de resposta imunológica humoral em coelhos. A resposta induzida pelas imunizações foi avaliada quantitativamente, através da técnica ensaio imunoenzimático (ELISA) para a detecção de imunoglobulinas da classe $\mathrm{G}$ (IgG). A técnica do ELISA tem sido descrita como um método eficiente para detecção de anticorpos anti $\boldsymbol{P}$. insidiosum em equinos e coelhos (MENDOZA et al., 1997; LEAL et al., 2001).

\section{MATERIAL E MÉTODOS}

\section{Antígeno e adjuvantes}

$\mathrm{O}$ antígeno utilizado para imunização dos coelhos foi preparado a partir de culturas puras de $\boldsymbol{P}$. insidiosum (DMVP 118/98 - CBS 101555). As 
amostras foram cultivadas em caldo Sabouraud a $37^{\circ} \mathrm{C}$ sob agitação (150rpm) durante 6 a 7 dias. Após esse período, o cultivo foi filtrado e a massa micelial foi ressuspendida em PBS e sonicada até total rompimento das hifas, seguido de centrifugação (7000rpm / 5min) e coleta do sobrenadante para uso como antígeno. Esse antígeno foi denominado de antígeno macerado de micélio (AMM). O AMM possuía concentração de proteínas totais entre 2,5 e $3 \mathrm{mg} / \mathrm{m} \ell$. Os adjuvantes avaliados foram: hidróxido de alumínio ${ }^{\mathrm{a}}$, óleo mineral ${ }^{\mathrm{a}}$ e adjuvante de Freund ${ }^{\mathrm{b}}$. Como controle foi utilizada água destilada estéril.

\section{Animais e imunizações:}

Foram utilizados 24 coelhos saudáveis com idade de três a quatro meses, de ambos os sexos, das raças Chinchila e Nova Zelândia. Os animais foram divididos aleatoriamente em quatro grupos e a cada grupo foi designado um tratamento: grupo I- hidróxido de alumínio; grupo II- óleo mineral; grupo III- Freund; grupo IV- controle. Em cada imunização, cada coelho recebeu $0,5 \mathrm{~m} \ell$ de AMM (@1,4mg de proteína) associado a $0,5 \mathrm{~m} \ell$ de adjuvante ou água destilada estéril. Para uma perfeita homogeneização da mistura, foi realizada uma emulsão dos adjuvantes com o AMM. No grupo II foi utilizado o adjuvante completo de Freund na primeira imunização e o adjuvante incompleto de Freund nas demais aplicações. Durante o período experimental (120 dias), os coelhos mantidos em tratamento receberam 7 doses de imunógeno com intervalos de 14 dias e foram submetidos a coletas de sangue semanais. $\mathrm{O}$ soro obtido nessas coletas foi utilizado para detecção de anticorpos (IgG) antipythium. As imunizações foram realizadas pela via subcutânea na região costal. O dia da primeira imunização foi estabelecido como dia zero e o soro resultante da coleta inicial foi considerado soro préimune.

\section{Delineamento experimental}

$\mathrm{O}$ experimento foi dividido em duas fases. $\mathrm{Na}$ primeira fase, todos os coelhos receberam três doses de imunógeno (dias 0, 14 e 28). A partir do $42^{\circ}$ dia, iniciou-se a segunda fase, quando cada grupo foi subdividido em dois: destes, três coelhos foram mantidos em tratamento (imunizações adicionais nos dias 42, 56, 68 e 82), e os outros três tiveram o tratamento interrompido. Os subgrupos com tratamento prolongado receberam sete doses do imunógeno no total, enquanto os subgrupos com tratamento interrompido receberam apenas as três doses da fase 1 .

\section{Resposta sorológica}

A resposta sorológica às imunizações foi avaliada através do ensaio imunoenzimático em fase sólida (ELISA) indireto (SANTURIO et al., 2001). Placas de 96 cavidades contendo $10 \mu \mathrm{g} /$ cavidade de antígenos de $\boldsymbol{P}$. insidiosum foram incubadas com uma diluição dos soros (1:2.000) a serem testados. As placas foram incubadas por $1 \mathrm{~h}$ à $37^{\circ} \mathrm{C}$, seguido de aspiração das amostras. Após, as placas foram lavadas com PBS e incubadas com anticorpo antiIgG de coelho conjugado com peroxidase ${ }^{\mathrm{b}}$ $(1: 10.000)$ e novamente incubado por $1 \mathrm{~h}$ à $37^{\circ} \mathrm{C}$. A seguir, as placas foram novamente lavadas e incubadas com o substrato cromogênico (orthophenylene-diamine/ $\mathrm{OPD}^{\mathrm{c}}$ ) por 15 minutos. A reação foi bloqueada com $\mathrm{H}_{2} \mathrm{SO}_{4} 4 \mathrm{~N}$. A leitura foi realizada em um espectrofotômetro para microplacas, com filtro de 490nm.

Para a comparação dos níveis de anticorpos induzidos por cada tratamento, foram avaliadas as amostras dos 24 coelhos coletadas durante o experimento. $\mathrm{Na}$ primeira fase, foram avaliados os dias 14, 21, 28 e 35. Na segunda fase, cada subgrupo foi composto de três coelhos. A comparação dos coelhos com tratamento prolongado (fase 2) foi nos dias 42, 49, 62, 75, 82, 89 e 120 e a comparação daqueles com tratamento interrompido (fase 2) utilizou os soros coletados nos dias 42, 49, 62, 75 e 82. Uma amostra de soro de coelho não imunizado (sabidamente negativo) e uma de soro hiperimune foram usadas, respectivamente como controle negativo e controle positivo do teste. Para análise dos resultados foram utilizados os valores de densidade óptica (DO) resultante do teste ELISA. Os resultados (DO) foram submetidos à análise de variância, aplicando-se o teste Tukey $(\mathrm{P}<0,05)$ pelo programa estatístico GraphPad InStat ${ }^{\mathrm{d}}$ (1994).

\section{RESULTADOS}

A resposta humoral (IgG) anti-pythium nos diferentes grupos foi quantificada através da leitura de densidades óticas resultantes do ELISA, realizado com as amostras de soro obtidas nas diferentes datas. Os resultados foram comparados entre si, em cada data indicada, e submetidos à análise de variância, utilizando o teste Tukey $(\mathrm{p}<0,05)$. Na tabela 1 , são apresentados os resultados da comparação entre tratamentos durante a fase 1 (dias 14, 21, 28 e 35). Todos os coelhos foram negativos no dia zero, comprovando a especificidade da reação sorológica dos animais imunizados. No dia 14, o grupo III (óleo mineral) apresentou o maior nível de IgG, sendo estatisticamente superior aos demais grupos. O 
Tabela 1 - Resposta sorológica (IgG) de coelhos imunizados com Antígeno Macerado de Micélio (AMM) associado a diferentes adjuvantes, durante a fase 1 , avaliada através de ELISA*.

\begin{tabular}{ccccc}
\hline & \multicolumn{3}{c}{ Densidade (média)** } & \\
\cline { 2 - 4 } Adjuvante (grupo) & Dia 14 & Dia 21 & Dia 28 & Dia 35 \\
\hline Hidróxido de Al (G I) & $0,114^{\mathrm{a}}$ & $0,374^{\mathrm{a}}$ & $0,352^{\mathrm{a}}$ & $0,445^{\mathrm{a}}$ \\
Adj. de Freund (G II) & $0,285^{\mathrm{b}}$ & $0,508^{\mathrm{b}}$ & $0,529^{\mathrm{b}}$ & $0,594^{\mathrm{b}}$ \\
Óleo mineral (G III) & $0,424^{\mathrm{c}}$ & $0,466^{\mathrm{b}}$ & $0,455^{\mathrm{c}}$ & $0,500^{\mathrm{c}}$ \\
Controle (G IV) & $0,066^{\mathrm{a}}$ & $0,191^{\mathrm{c}}$ & $0,167^{\mathrm{d}}$ & $0,225^{\mathrm{d}}$ \\
Média final & 0,222 & 0,384 & 0,375 & 0,441 \\
F & 61,85 & 43,34 & 109,01 & 159,81 \\
P< & 0,01 & 0,05 & 0,05 & 0,05 \\
\hline
\end{tabular}

* Densidade ótica (DO), medida a 490nm.

** Letras diferentes na mesma coluna representam diferenças estatisticamente significativas $(\mathrm{P}<0,05)$.

grupo II (adjuvante de Freund) foi estatisticamente superior ao grupo I (hidróxido de alumínio) e ao grupo IV (controle), enquanto esses últimos não apresentaram diferença entre si. Nos dias 21,28 e 35 os três grupos de adjuvantes apresentaram diferença estatística em relação ao grupo controle. Dentre os adjuvantes, os oleosos (II e III) foram superiores ao grupo I durante toda fase 1 . Nos dias 28 e 35 , os grupos II e III apresentaram diferenças estatísticas entre si.

$\mathrm{Na}$ fase 2, os animais que permaneceram em tratamento receberam um total de sete doses de imunógeno, sendo a última dose aplicada no $82^{\circ}$ dia. Entre os dias 42 e 120, foram realizadas sete avaliações no nível de anticorpos. Os resultados dessa avaliação estão apresentados na tabela 2. Em todas as datas avaliadas, os grupos imunizados com adjuvantes apresentaram resultados estatisticamente superior ao grupo controle. $\mathrm{O}$ grupo II apresentou as maiores médias de DO durante a fase 2, porém apenas nos dias 42, 49 e 120 foi estatisticamente superior aos grupos I e III. Nos grupos I e III, não foram observadas diferenças estatísticas nos coelhos mantidos em tratamento durante a fase 2 .

A comparação entre os animais que tiveram o tratamento interrompido utilizou amostras de soro coletadas nos dias 42, 49, 62, 75 e 82 (fase 2). O resumo das avaliações sorológicas desses animais são apresentados na tabela 3. Como no subgrupo anterior, os coelhos dos grupos I, II e III apresentaram níveis de anticorpos estatisticamente superiores ao observado no grupo controle. O adjuvante de Freund foi estatisticamente superior ao hidróxido de alumínio em todas as datas (fase 2). Não foram observadas diferenças significativas entre os adjuventes oleosos (II e III). O óleo mineral foi superior ao hidróxido de alumínio apenas no dia 75. Os resultados do subgrupo com tratamento interrompido evidenciaram uma redução nos níveis de anticorpos do grupo controle no decorrer da fase 2 , enquanto os tratados com adjuvantes apresentaram manutenção dos níveis.

\section{DISCUSSÃO}

O desenvolvimento de vacinas contra organismos eucariotas representa um desafio, pois a complexidade antigênica desses organismos dificulta a seleção de componentes imunogênicos com potencial protetor. Nos últimos anos, com o aumento da ocorrência das doenças fúngicas associadas a enfermidades imunossupressoras, grandes esforços têm sido realizados para o desenvolvimento de imunógenos para a prevenção e tratamento dessas infecções. Doenças micóticas causadas por fungos como Aspergillus fumigatus, Candida albicans, Cryptococcus neoformans, Trichophyton sp, Blastomyces dermatidis, Histoplasma capsulatum, Paracoccidioides brasiliensis e Pythium insidiosum estão sob investigação e já demonstraram o potencial

Tabela 2 - Resposta sorológica (IgG) de coelhos imunizados com Antígeno Macerado de Micélio (AMM) associado a diferentes adjuvantes, durante a fase 2, coelhos mantidos em tratamento, avaliada através de ELISA*

\begin{tabular}{cccccccc}
\hline & & \multicolumn{3}{c}{ Densidade (média)** } & \\
\cline { 4 - 6 } Adjuvante (grupo) & Dia 42 & Dia 49 & Dia 62 & Dia 75 & Dia 82 & Dia 89 & Dia 120 \\
\hline Hidróxido Al (G I) & $0,406^{\mathrm{a}}$ & $0,461^{\mathrm{a}}$ & $0,489^{\mathrm{ab}}$ & $0,524^{\mathrm{ab}}$ & $0,528^{\mathrm{ab}}$ & $0,527^{\mathrm{ab}}$ & $0,396^{\mathrm{a}}$ \\
Adj. Freund (G II) & $0,602^{\mathrm{b}}$ & $0,611^{\mathrm{b}}$ & $0,624^{\mathrm{a}}$ & $0,625^{\mathrm{a}}$ & $0,620^{\mathrm{a}}$ & $0,637^{\mathrm{a}}$ & $0,607^{\mathrm{b}}$ \\
Óleo mineral (G III) & $0,472^{\mathrm{a}}$ & $0,487^{\mathrm{a}}$ & $0,475^{\mathrm{b}}$ & $0,423^{\mathrm{b}}$ & $0,422^{\mathrm{b}}$ & $0,450^{\mathrm{b}}$ & $0,451^{\mathrm{a}}$ \\
Controle (G IV) & $0,234^{\mathrm{c}}$ & $0,234^{\mathrm{c}}$ & $0,251^{\mathrm{c}}$ & $0,240^{\mathrm{c}}$ & $0,204^{\mathrm{c}}$ & $0,203^{\mathrm{c}}$ & $0,126^{\mathrm{c}}$ \\
Média final & 0,428 & 0,448 & 0,459 & 0,411 & 0,443 & 0,454 & 0,395 \\
F & 127,65 & 55,86 & 36,43 & 14,54 & 49,55 & 50,96 & 153,28 \\
P< & 0,01 & 0,05 & 0,05 & 0,01 & 0,01 & 0,01 & 0,01 \\
\hline
\end{tabular}

\footnotetext{
* Densidade ótica (DO), medida a 490nm.

** Letras diferentes na mesma coluna representam diferenças estatisticamente significativas $(\mathrm{P}<0,05)$.
} 
Resposta sorológica de coelhos imunizados com antígenos de Pythium insidiosum ...

Tabela 3 - Resposta sorológica (IgG) de coelhos imunizados com Antígeno Macerado de Micélio (AMM) associado a diferentes adjuvantes, durante a fase 2, coelhos com tratamento interrompido, avaliada através de ELISA*.

\begin{tabular}{|c|c|c|c|c|c|}
\hline \multirow[b]{2}{*}{ Adjuvante (grupo) } & \multirow[b]{2}{*}{ Dia 42} & \multicolumn{2}{|c|}{ Densidade (média)** } & \multirow[b]{2}{*}{ Dia 75} & \multirow[b]{2}{*}{ Dia 82} \\
\hline & & Dia 49 & Dia 62 & & \\
\hline Hidróxido de $\mathrm{Al}$ (G I) & $0,430^{\mathrm{a}}$ & $0,419^{\mathrm{a}}$ & $0,432^{\mathrm{a}}$ & $0,413^{\mathrm{a}}$ & $0,423^{\mathrm{a}}$ \\
\hline Adj. Freund (G II) & $0,589^{b}$ & $0,596^{\mathrm{b}}$ & $0,611^{\mathrm{b}}$ & $0,585^{\mathrm{b}}$ & $0,626^{\mathrm{b}}$ \\
\hline Óleo mineral (G III) & $0,519^{\mathrm{ab}}$ & $0,519^{\mathrm{ab}}$ & $0,469^{\mathrm{ab}}$ & $0,527^{\mathrm{b}}$ & $0,474^{\text {ab }}$ \\
\hline Controle (G IV) & $0,249^{\mathrm{c}}$ & $0,234^{\mathrm{c}}$ & $0,197^{\mathrm{c}}$ & $0,189^{\mathrm{c}}$ & $0,129^{\mathrm{c}}$ \\
\hline Média final & 0,446 & 0,442 & 0,427 & 0,428 & 0,413 \\
\hline $\mathrm{F}$ & 58,41 & 50,51 & 38,88 & 563,05 & 23,90 \\
\hline $\mathrm{P}<$ & 0,01 & 0,01 & 0,05 & 0,01 & 0,05 \\
\hline
\end{tabular}

* Densidade ótica (DO), medida a 490nm.

** Letras diferentes na mesma coluna representam diferenças estatisticamente significativas $(\mathrm{P}<0,05)$.

das vacinas (NEWTON \& ROSS, 1993; DIXON et al., 1998). Em alguns casos, já foram identificados antígenos protetores, como a gp43 do $\boldsymbol{P}$. brasiliensis, que atualmente está sendo estudada como vacina de DNA (PINTO et al., 2000). No caso do $\boldsymbol{P}$. insidiosum, já foram identificados alguns antígenos imunodominantes potencialmente protetores, sem no entanto, obter-se a comprovação dos mecanismos imunológicos desencadeados por tais antígenos (MENDOZA et al., 1992b; LEAL, 1999).

O presente estudo objetivou avaliar o efeito imunogênico de antígenos de $\boldsymbol{P}$. insidiosum associados a diferentes adjuvantes, servindo de modelo para potencial aplicação em eqüinos. A comparação entre tratamentos permitiu identificar diferenças significativas com alto grau de confiança $(\mathrm{P} \leq 0,05)$. Entre os grupos avaliados, os adjuvantes oleosos (grupos II e III) induziram resposta sorológica de magnitude estatisticamente superior à induzida pelo hidróxido de alumínio e pelo antígeno sem adjuvante (controle) durante a fase 1. Nos subgrupos mantidos em tratamento (fase 2), todos os adjuvantes foram superiores ao grupo controle. $\mathrm{O}$ adjuvante de Freund apresentou as maiores médias, porém, apenas em algumas datas, a diferença foi estatisticamente superior. No início do tratamento, o óleo mineral foi superior ao hidróxido de alumínio. Essa diferença, no entanto, foi reduzindo-se no decorrer das imunizações, com o hidróxido de alumínio apresentando médias de DO superiores entre os dias 62 e 89 . No subgrupo com tratamento interrompido (fase 2), observou-se um declínio progressivo nos níveis de anticorpos do grupo controle, enquanto nos grupos com adjuvantes, os níveis mantiveram-se relativamente estáveis. Isso também foi observado após o final das imunizações no subgrupo mantido em tratamento (dia 120).

Quanto aos adjuvantes testados, o adjuvante de Freund apresentou os melhores resultados, seguido do óleo mineral e do hidróxido de alumínio. Contudo, o adjuvante de Freund é indicado apenas para estudos experimentais, não sendo permitido seu uso comercial. O óleo mineral, estatisticamente superior ao hidróxido de alumínio na fase 1 , seria o primeiro candidato para avaliação em animais com pitiose. De acordo com KLEIN \& HOREJSI (1997), entre as principais ações dos adjuvantes destacamse: 1- formação de depósito; 2- estimulação da migração celular; 3- aumento da dispersão; 4estímulo na proliferação de linfócitos; 5- auxílio à mensageiros químicos; 6- podem aumentar a probabilidade de contato entre células $\mathrm{T}, \mathrm{B}$, macrófagos e antígenos; 7- podem inclinar o balanço entre tolerância e imunidade em favor do último.

Essa eficiência dos adjuvantes em potencializar a resposta imunológica é amplamente conhecida, porém o efeito dessas substâncias pode variar de acordo com os antígenos envolvidos. $\mathrm{O}$ presente estudo demonstrou um aumento significativo na intensidade e na duração da resposta humoral induzida por antígenos de $\boldsymbol{P}$. insidiosum associado aos adjuvantes testados. Esses resultados indicam a possibilidade de aumento nos índices de cura pelo uso do imunoterápico, quando esse é associado a adjuvantes. Nesse estudo, avaliou-se apenas a resposta humoral representada por IgG. Embora possa ser considerado um indicador da magnitude da resposta imunológica, a quantificação de IgG sérica não pode ser tomada como parâmetro definitivo na avaliação da resposta imunológica ao Pythium insidiosum. Resposta mediada por células (linfócitos CD4+, mastócitos, macrófagos e eosinófilos), assim como resposta humoral por $\operatorname{IgE}$ têm demonstrado ser importantes na resposta imunológica aos fungos (KLEIN \& HOREJSI, 1997; TIZARD, 1996; DEEPE \& BULLOCK, 1990) e provavelmente desempenham papel relevante na cura induzida pela imunoterapia. Por outro lado, a manutenção de níveis detectáveis de IgG por um período prolongado permite projetar a utilização do imunoterápico também como preventivo. 


\section{FONTES DE AQUISIÇÃO}

${ }^{a}$ IRFA, Porto Alegre - RS - Brasil.

${ }^{\mathrm{b}}$ Sigma Chemical Co, St. Louis, Missouri - USA.

'Pierce, Rocford, IL - USA.

${ }^{\mathrm{d}}$ GraphPad Software, San Diego - USA.

\section{REFERÊNCIAS BIBLIOGRÁFICAS}

ALEXOPOULOS, C.J., MIMS, C.W., BLACKWELL, M. Introductory mycology. 4. ed. New York : John Wiley \& Sons, 1996. Cap 23: Phylum Oomycota: p.683-737.

CHAFFIN, M.K., SCHUMACHER, J., McMULLAN, W.C. Cutaneous pythiosis in the horse. Vet Clin North Am: Equine Pract, v.11, n1, p.91-103, 1995.

DEEPE, G S., BULLOCK, W.E. Immunological aspects of fungal pathogenesis. Europ J Clin Microbiol Infect Dis, v.9, n.8, p 567-579, 1990

DIXON, D.M., CASADEVAlL, A., KLEIN, B., et al. Development of vaccines and their use in prevention of fungal infections. Medical Mycology, v.36, p.57-67, 1998.

KLEIN, J., HOREJSI, V. Immunology. 2 ed. London : Blackwell Science, 1997. 722 p. Cap.13: Antigens: p.393421. Cap.21: Defense against invaders: p. 532-595.

FOIL, C.S. Update on pythiosis (Oomycosis). In: THE NORTH AMERICAN VETERINARY CONFERENCE, 1996, Orlando. Proceedings... Orlando : Bayer Animal Health, 1996. p.57-63.

LEAL, A.T., LEAL, A.B.M., FLORES, E.F., et al. Pitiose Ciência Rural, v.31, n.4, p.735-743, 2001.

LEAL, A.T. Pythium insidiosum: caracterização antigênica preliminar e avaliação de adjuvantes na indução de resposta sorológica em coelhos. Santa Maria, 1999. 94 p. Dissertação (Mestrado em Medicina Veterinária) - Programa de Pós-graduação em Medicina Veterinária, Universidade Federal de Santa Maria, 1999.

MEIRELES, M.C.A., RIET-CORREA, F., FISCHMAN, O., et al. Cutaneous pythiosis in horses from Brazil. Mycoses, v.36, p.139-142, 1993.

MENDOZA, L., ALFARO, A.A. Equine pythiosis in Costa Rica: Report of 39 cases. Mycopathologia, v.94, p.123-129, 1986.
MENDOZA, L., AJELLO, L., McGINNIS, M.R. Infections caused by the oomycetous pathogen Pythium insidiosum. J Mycol Med, v.6, n.4, p.151-164, 1996.

MENDOZA, L., NICHOLSON, V., PRESCOTT, J.F. Immunoblot analysis of the humoral immune response to Pythium insidiosum in horses with pythiosis. J Clin Microbiol, v.30, n.11, p.2980-2983, 1992b.

MENDOZA, L., VILlalobOS, J., CALlEJA, C. E., et al. Evaluation of two vaccines for the treatment of pythiosis insidiosi in horses. Mycopathologia, v.119, p.89-95, 1992a.

MENDOZA, L., KAUFMAN, L., MANDY, W. $\boldsymbol{e}$ t al. Serodiagnosis of human and animal pythiosis using na enzyme-linked immunosorbent assay. Clin Diagn Lab Immunol, v.4, n.6, p.715-718, 1997.

MILLER, RI. Treatment of equine phycomycosis by immunotherapy and surgery. Aust Vet J, v. 57, p.377-382, 1981.

MILLER, R.I., CAMPBELL, R.S.F. Clinical observations on equine phycomycosis. Aust Vet J, v.58, p.221-226, 1982.

MILLER, R.I., CAMPBELL, R.S.F. Experimental pythiosis in rabbits. Sabouraudia, v.21, p.331-341, 1983.

MONTEIRO, A.B. Imunoterapia da pitiose eqüina: teste de eficácia de um imunobiológico e avaliação leucocitária em animais infectados naturalmente pelo Pythium insidiosum. Santa Maria, 1999. 52 p. Dissertação (Mestrado em Medicina Veterinária) - Programa de Pós-graduação em Medicina Veterinária, Universidade Federal de Santa Maria, 1999.

NEWTON, J.C., ROSS, P.S. Equine pythiosis: an overview of immunotherapy. Compend Contin Educ Pract Vet, v.15, n.3, p.491-493, 1993.

PINTO, A.R., PUCCIA, R., DINIZ, S.N., et al. DNA-based vaccination against murine paracoccidioidomycosis using the gp43 gene from Paracoccidioides brasiliensis. Vaccine, v.18, n.26, p. 050-3058, 2000

SANTURIO, J.M., LEAL, A.T., LEAL, A.B.M., et al. Teste ELISA para o diagnóstico de pitiose. In: CONGRESSO BRASILEIRO DE MICOLOGIA, 3, 2001, Aguas de Lindóia, SP. Anais... Águas de Lindóia : Sociedade Brasileira de Micologia, 2001. p.126.

TIZARD, I.R. Veterinary immunology - An introduction. 5 ed. Philadelphia, Pennsylvania : Saunders, 1996. 531p.

VANSELOW, B.A. The application of adjuvants to veterinary medicine. Veterinary Bulletin, v.57, n.11, p.881-896, 1987. 